\title{
A Form of Adsorptive Pre-wave in a Sampled $d c$ Polarography on a Static Mercury Drop Electrode
}

\author{
Šebojka Komorsky-Lovrić, ${ }^{*}$ Milivoj Lovrić \\ Center for Marine and Environmental Research, "Rudjer Bošković" Institute, \\ P.O. Box 180, 10002 Zagreb, Croatia
}

Received 13 October 2005; accepted 3 January 2006

\begin{abstract}
Theoretical analyses show that in a sampled $d c$ polarography on the static mercury drop electrode the response may consist of a pre-wave and a main wave that are separated by a minimum. This is a general characteristic of electrode reactions complicated by strong adsorption of the product. A steep pre-wave and the minimum are the indications of Frumkin isotherm. A flat plateau of the pre-wave is a special case of the steep plateau which appears at lower reactant concentrations. If the adsorption is weak, the pre-wave and the main wave are usually merged into a single wave.
\end{abstract}

Keywords: polarography, adsorption, mercury electrode.

\section{Introduction}

If a reactant of the reversible electrode reaction is adsorbed on the dropping mercury electrode surface, a $d c$ polarogram may consist of a main wave and adsorptive post-wave. If only the product is adsorbed, the adsorptive pre-wave and the main wave may appear $[1,2]$. However, in the sampled $d c$ polarography using a static mercury drop electrode, the main wave and the post-wave may be separated by a deep and narrow minimum [3-5]. This phenomenon was observed in experiments with lead ions in bromide medium [3]. It is caused by the adsorption of neutral complex $\mathrm{PbBr}_{2}$ [4]. The minimum is the indication of lateral attractions in the adsorbed layer [5]. In the present communication, the possibility that the adsorption pre-wave and the diffusion main wave are also separated by the minimum is demonstrated theoretically, and the origin of the minimum is investigated.

\footnotetext{
* Corresponding author. E-mail address: slovric@rudjer.irb.hr
} 


\section{The model}

A reversible redox reaction complicated by the adsorption of product on the surface of the static mercury drop electrode is considered:

$$
\mathrm{Ox}+\mathrm{ne}^{-} \leftrightarrows \mathrm{Red} \leftrightarrows(\mathrm{Red})_{\mathrm{ads}}
$$

The mass transfer is described by the semi-infinitive planar diffusion model:

$$
\begin{gathered}
\frac{\partial c_{o x}}{\partial t}=D \frac{\partial^{2} c_{o x}}{\partial x^{2}} \\
\frac{\partial c_{r e d}}{\partial t}=D \frac{\partial^{2} c_{r e d}}{\partial x^{2}} \\
t=0, \quad x \geq 0: \quad c_{o x}=c_{o x}^{*}, \quad c_{r e d}=0, \quad \Gamma_{r e d}=0 \\
t>0, \quad x \rightarrow \infty: \quad c_{o x} \rightarrow c_{o x}^{*}, \quad c_{r e d} \rightarrow 0 \\
x=0: \quad\left(c_{o x}\right)_{x=0}=\left(c_{r e d}\right)_{x=0} \cdot \exp (\varphi) \\
\varphi=n F\left(E-E^{0}\right) / R T \\
\beta\left(c_{r e d}\right)_{x=0} \cdot \exp (-a \theta)=\theta /(1-\theta) \\
\theta=\Gamma_{r e d} / \Gamma_{\max } \\
D\left(\partial c_{o x} / \partial x\right)_{x=0}=I / n F S \\
D\left(\partial c_{r e d} / \partial x\right)_{x=0}=-I / n F S+d \Gamma_{r e d} / d t
\end{gathered}
$$

The meanings of all symbols are given in the Table 1. This model applies as the first approximation if the drop life-time is shorter than three seconds [6].

Table 1

\begin{tabular}{|l|l|}
\hline$a$ - Frumkin coefficient of the isotherm & $R$ - Gas constant \\
\hline $\begin{array}{l}c_{o x}, c_{r e d} \text { - Concentrations of the reactant and } \\
\text { the product, respectively }\end{array}$ & $S$ - Electrode surface area \\
\hline$c_{o x}^{*}$ - Bulk concentration of the reactant & $T$ - Time \\
\hline$D$ - Diffusion coefficient & $t_{d}$ - Drop life-time in $d c$ polarography \\
\hline$E$ - Electrode potential & $T$ - Temperature \\
\hline$E^{0}$ - Standard potential & $\beta$ - Adsorption constant \\
\hline$F$ - Faraday constant & $\Gamma_{r e d}$ - Surface concentration of the adsorbed product \\
\hline$I$ - Current & $\begin{array}{l}\Gamma_{\text {max }} \text { - The maximum surface concentration of the } \\
\text { adsorbed product }\end{array}$ \\
\hline$n$ - Number of electrons & \\
\hline
\end{tabular}


Eq. (1) and (2) are solved by the substitution $\psi=c_{o x}+c_{r e d}$ [3]. The solution is a system of recursive formulae for the degree of coverage of the electrode surface:

$$
\left(\theta_{m}-1\right)\left(\theta_{m}-\omega_{m}\right)=y \cdot \theta_{m} \cdot \exp \left(a \theta_{m}\right)
$$

where $\theta_{m}$ is the surface coverage in the moment $t=m d$ and $d$ is the time increment, $m=1,2, \ldots . M$ and $M=t_{d} / d$. The meanings of the other symbols are as follows:

$$
\begin{gathered}
\omega_{m}=\frac{p \sqrt{\pi}}{2 \sqrt{M}}-\sum_{j=1}^{m-1} \theta_{j}\left(S_{m-j+1}-S_{m-j}\right) \\
y=\frac{\sqrt{\pi}}{2 q \sqrt{M}}[1+\exp (\varphi)] \\
p=\frac{c_{o x}^{*} \sqrt{D \cdot t_{d}}}{\Gamma_{\max }} \\
q=\frac{\beta \cdot \Gamma_{\max }}{\sqrt{D \cdot t_{d}}} \\
S_{k}=\sqrt{k}-\sqrt{k-1}
\end{gathered}
$$

Eq. (3) is solved by a numerical search within the interval $0 \leq \theta_{m} \leq 1$ [5]. The simulation was performed by using the number of time increments $M=1000$. The current was calculated by solving eq. (1):

$$
\frac{I_{m}}{I_{L}}=\sqrt{\frac{M}{m}}-2 \sqrt{M}\left[\frac{\left(c_{o x}\right)_{x=0, m}}{c_{o x}^{*}}+\sum_{j=1}^{m-1} \frac{\left(c_{o x}\right)_{x=0, j}}{c_{o x}^{*}}\left(S_{m-j+1}-S_{m-j}\right)\right]
$$

where: $I_{L}=n F S c_{o x}^{*} \sqrt{D / \pi \cdot t_{d}}$ and $\frac{\left(c_{o x}\right)_{x=0, j}}{c_{o x}^{*}}=\frac{1}{p \cdot q} \cdot \frac{\theta_{j}}{1-\theta_{j}} \cdot \exp (\varphi) \cdot \exp \left(a \theta_{j}\right)$.

In the sampled $d c$ polarography, the current is measured at the end of each drop $(m=M)$, but the change of the surface coverage and the current during the $95 \%$ of the life-time of a single drop $(50 \leq m \leq M)$ were also simulated.

The concentration profiles of the reactant were calculated by using the following equation:

$$
\begin{aligned}
& \frac{c_{o x, m}}{c_{o x}^{*}}=\operatorname{erf}\left(\frac{x \sqrt{M}}{2 \sqrt{D \cdot t_{d}} \sqrt{m}}\right)+\frac{\left(c_{o x}\right)_{x=0, m}}{c_{o x}^{*}} \cdot \operatorname{erfc}\left(\frac{x \sqrt{M}}{2 \sqrt{D \cdot t_{d}}}\right) \\
& +\sum_{j=1}^{m-1} \frac{\left(c_{o x}\right)_{x=0, j}}{c_{o x}^{*}}\left[\operatorname{erf}\left(\frac{x \sqrt{M}}{2 \sqrt{D \cdot t_{d}} \sqrt{m-j}}\right)-\operatorname{erf}\left(\frac{x \sqrt{M}}{2 \sqrt{D \cdot t_{d}} \sqrt{m-j+1}}\right)\right]
\end{aligned}
$$




\section{Results and discussion}

The sampled $d c$ polarogram of electrode reaction (I) depends on the dimensionless bulk concentration of the reactant $p=c_{o x}^{*}\left(D \cdot t_{d}\right)^{1 / 2} / \Gamma_{\max }$, the dimensionless adsorption constant of linear isotherm $q=\beta \cdot \Gamma_{\max }\left(D \cdot t_{d}\right)^{-1 / 2}$ and the Frumkin coefficient $a$. For $q=100$ and $a=-3$, the influence of the parameter $p$ is shown in Fig. 1. If $p<0.5$, only a pre-wave appears because the electrode surface is not fully covered by the adsorbed product before the end of the drop life-time. In the narrow range $0.5<p<0.9$, the pre-wave and the main wave are unresolved, but for $p>0.9$ these two waves are separated by a minimum.

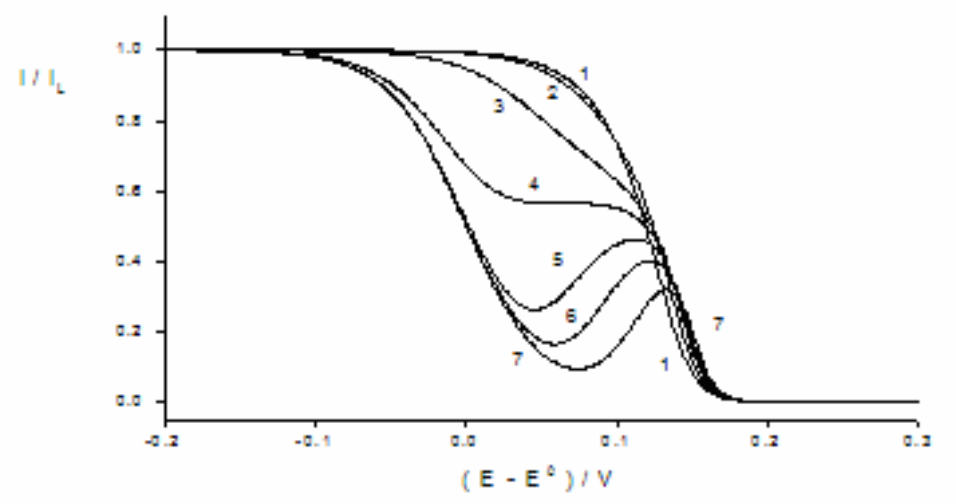

Figure 1. Dependence of dimensionless sampled $d c$ polarograms on the dimensionless bulk concentration of the reactant; $q=100, a=-3$ and $p=0.5$ (1), 0.7 (2), 0.85 (3), 0.9 (4), 0.95 (5), 1 (6) and 1.1 (7).

Fig. 2 shows the influence of the adsorption constant $q$ for the Langmuir isotherm. If the value of $q$ is increased, the pre-wave develops at higher potentials and the minimum appears eventually.

Under the influence of lateral attractions in the adsorbed layer, the pre-wave is steeper and its separation from the main wave is improved. This is shown in Fig. 3. Comparing the curve 2 in Fig. 2 with the curves in Figs. 1 and 3, it can be noticed that the potential at which the pre-wave develops depends primarily on the parameter $q$, but the form of the pre-wave depends on the other two parameters. A well defined plateau of the pre-wave that can be seen in curve 4 in Figs. 1 and 3 disappears if the parameters $a$ and $p$ are either increased, or decreased.

The minimum is a consequence of the change of the concentration of the reactant at the electrode surface during the life-time of a single drop. In the case of a simple electrode reaction, without adsorption, this concentration is a sigmoidal function of the potential:

$$
\left(c_{o x}\right)_{x=0} / c_{o x}^{*}=\exp (\varphi) /[1+\exp (\varphi)]
$$




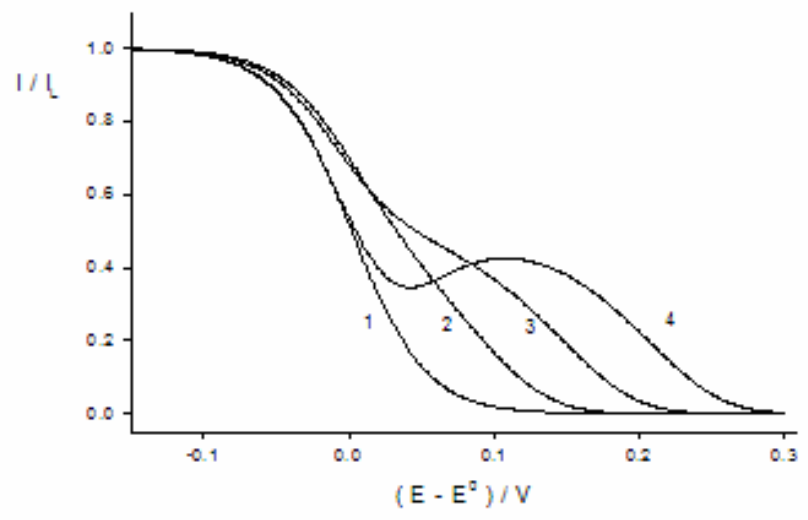

Figure 2. Dependence of dimensionless sampled $d c$ polarograms on the dimensionless adsorption constant of linear isotherm; $p=0.9, a=0$ and $q=1$ (1), 100 (2), 1000 (3) and 10000 (4).

As the potential changes in a stepwise manner from drop to drop, but not during the life-time of a single drop, the concentration $\left(c_{o x}\right)_{x=0}$ is independent of time. If the electrode reaction is complicated by the product adsorption which follows a linear isotherm:

$$
K\left(c_{\text {red }}\right)_{x=0}=\Gamma_{\text {red }}
$$

the concentration $\left(c_{o x}\right)_{x=0}$ is defined by the following equation:

$$
\frac{\left(c_{o x}\right)_{x=0}}{c_{o x}^{*}}=\frac{\exp (\varphi)}{1+\exp (\varphi)} \cdot\left[1-\exp \left(\frac{\{1+\exp (\varphi)\}^{2} D t}{K^{2}}\right) \cdot \operatorname{erfc}\left(\frac{\{1+\exp (\varphi)\} \sqrt{D t}}{K}\right)\right]
$$

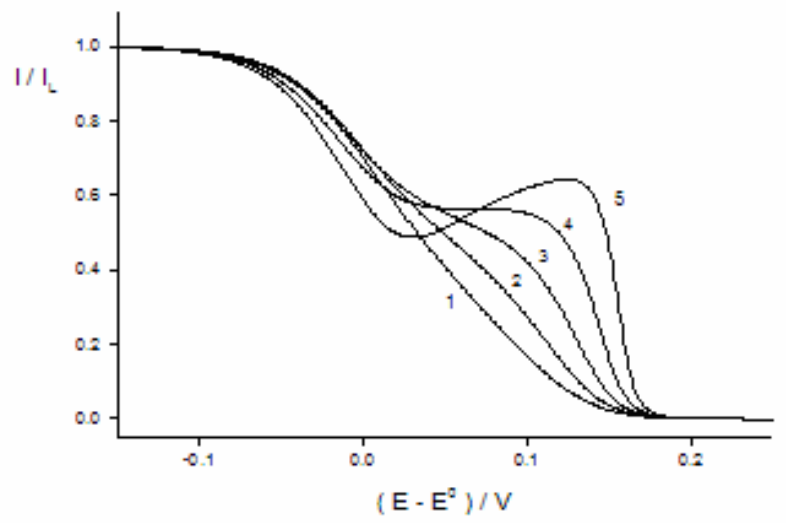

Figure 3. Dependence of dimensionless sampled $d c$ polarograms on the Frumkin coefficient; $p=0.9, q=100$ and $a=0$ (1), -1 (2), -2 (3), -3 (4) and -4 (5). 
The function in the square brackets changes from zero, for $t=0$, to 1 for $t \rightarrow \infty$. The concentration $\left(c_{o x}\right)_{x=0}$ decreases sigmoidally from $c_{o x}^{*}$ to zero in the potential range in which the pre-wave is developed. During a single drop, this concentration increases from nearly zero, at the beginning of the drop life-time, to $\left(\Gamma_{r e d}\right)_{t=t_{d}} \exp (\varphi) / K$ at the end of the drop life-time (see eq. 7 and the condition c). If the adsorption follows Frumkin isotherm, the change of $\left(c_{o x}\right)_{x=0}$ is more complex because of the saturation of electrode surface by the adsorbed product. This can be seen in Fig. 4. The dimensionless pre-wave exhibits a maximum at $0.122 \mathrm{~V}$ and a minimum at $0.059 \mathrm{~V}$, after which a main wave is developed. The concentration $\left(c_{o x}\right)_{x=0}$ decreases in two steps: the first one follows the development of the pre-wave and the second one the main wave. The second inflection point of the curve 2 appears at $0.090 \mathrm{~V}$. The surface coverage $\theta$ increases from 0.77 , at $0.122 \mathrm{~V}$, to 0.93 , at $0.090 \mathrm{~V}$, and to 0.98 at $0.059 \mathrm{~V}$. The stagnation of $\left(c_{o x}\right)_{x=0}$ at the step is caused by the fact that the decreasing of the factor $\exp (\varphi)$ is compensated by the increasing of the ratio $\theta /(1-\theta)$ (see below eq. 4). Fig. 4 is paradigmatic for all $p>0.9$, if $q=100$ and $a=-3$.

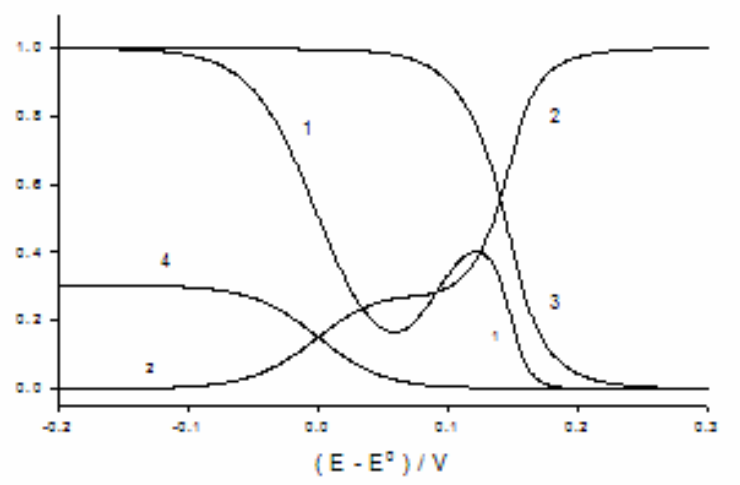

Figure 4. Dependence of dimensionless sampled $d c$ current, $I / I_{L}(1)$, dimensionless reactant concentration at the electrode surface, $\left(c_{o x}\right)_{x=0} / c_{o x}^{*}(2)$, the surface coverage, $\theta$ (3), and dimensionless product concentration at the electrode surface, $\left(c_{r e d}\right)_{x=0} / c_{o x}^{*}$ (4) on the electrode potential; $p=1, q=100$ and $a=-3$.

Fig. 5 shows the change of the current, the concentration $\left(c_{o x}\right)_{x=0}$ and the surface coverage during a life-time of a single drop at the potential of the minimum between the pre-wave and the main wave. In the last quarter of the life-time $\theta$ changes from 0.92 to 0.98 , the ratio $\theta \cdot \exp (a \theta) /(1-\theta)$ increases from 0.7 to 2.6 and the concentration $\left(c_{o x}\right)_{x=0}$ increases 3.5 times. As a consequence, the current decreases with time faster than predicted by the linear diffusion model. This is explained in Fig. 6 in which the reactant concentrations within the diffusion layer are shown. As $\left(c_{o x}\right)_{x=0}$ is increased, the gradient $\left(\partial c_{o x} / \partial x\right)_{x=0}$ is decreasing, which means that the current is diminished. 


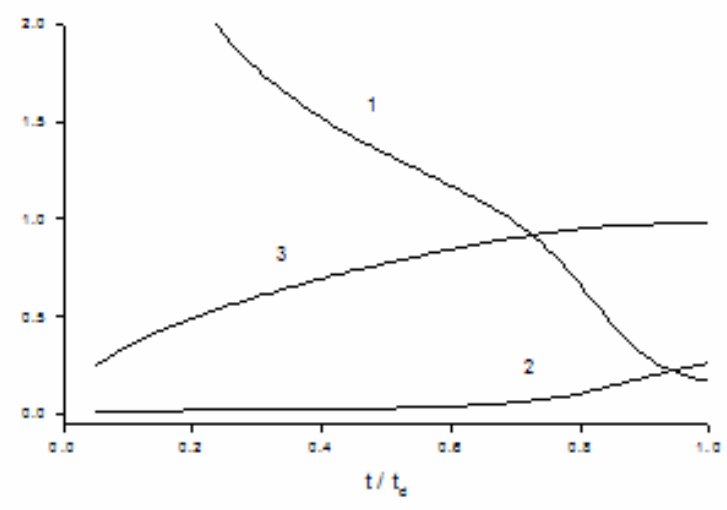

Figure 5. A change of $I / I_{L}(1),\left(c_{o x}\right)_{x=0} / c_{o x}^{*}$ (2) and $\theta$ (3) during the life-time of a single drop; $p=1, q=100, a=-3$ and $E-E^{0}=0.059 \mathrm{~V}$.

Fig. 7 shows a polarogram with a well defined plateau that separates the prewave from the main wave. The inflection point of the plateau appears at $0.070 \mathrm{~V}$. The surface coverage increases from 0.84 , at $0.1 \mathrm{~V}$, to 0.93 , at $0.070 \mathrm{~V}$, and to 0.98 at $0.030 \mathrm{~V}$, at which potential a main wave starts to develop. The dependence of $\left(c_{o x}\right)_{x=0}$ on the potential exhibits a tail, but not a step as in Fig. 4. This is because the increase of $\theta$ is slower if $p=0.9$ than if $p=1$ : in Fig. 7 the change from 0.93 to 0.98 occurs within $40 \mathrm{mV}$, while in Fig. 4 it occurs within $30 \mathrm{mV}$. So, for $p=0.9$, the decreasing of the factor $\exp (\varphi)$ is only partly compensated by the increasing of the ratio $\theta /(1-\theta)$. The second consequence is that at the plateau of the pre-wave the decreasing of current during the life-time of a single drop is less severe than in Fig. 5. This is shown in Fig. 8. The surface coverage increases only up to 0.93 and the corresponding increasing of $\left(c_{o x}\right)_{x=0}$ is rather small.

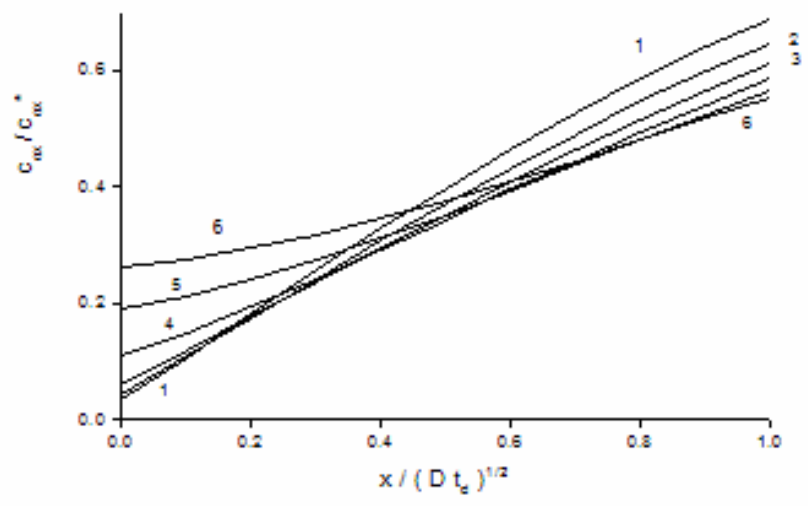

Figure 6. Dimensionless concentration of the reactant as a function of the dimensionless distance from the electrode surface during the life-time of a single drop; $p=1, q=100, a=-3, E-E^{0}=0.059 \mathrm{~V}$ and $t / t_{d}=0.5(1), 0.6(2), 0.7$ (3), 0.8 (4), 0.9 (5) and $1(6)$. 
If $p<0.9$, the gradient $d \theta / d E$ at the top of pre-wave is diminished and the plateau of pre-wave becomes steeper. So, the separation of the pre-wave from the main wave is reduced.

\section{Conclusion}

These results show that the minimum between the pre-wave and the main wave in the sampled $d c$ polarography on the static mercury drop electrode is a general characteristic of electrode reactions complicated by strong adsorption of the product. It appears at higher reactant concentrations as a consequence of the saturation of electrode surface. A steep pre-wave and the minimum are the indications of Frumkin isotherm.

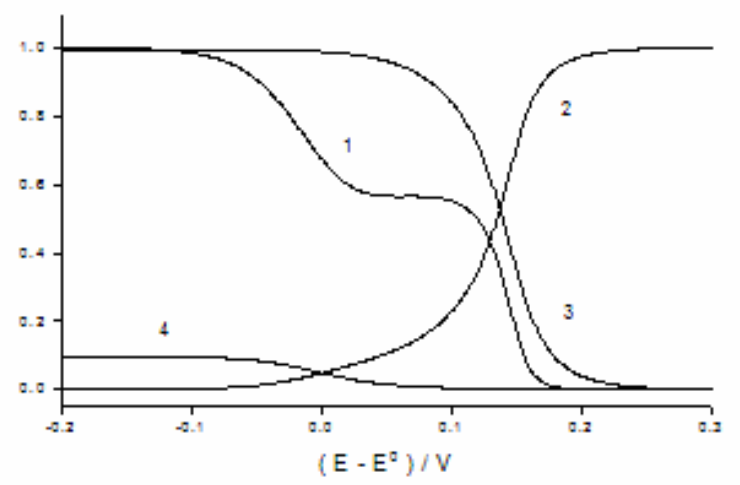

Figure 7. Dependence of $I / I_{L}(1),\left(c_{o x}\right)_{x=0} / c_{o x}^{*}(2), \theta$ (3) and $\left(c_{r e d}\right)_{x=0} / c_{o x}^{*}$ (4) on the electrode potential; $p=0.9, q=100$ and $a=-3$.

Usually, the adsorption is apparently strong if a moderate adsorption constant is enhanced by the lateral attractions in the adsorbed layer. In the less probable case the adsorption constant is very high, without interactions between adsorbed molecules. The pre-wave with a flat plateau may appear at a certain reactant concentration, but it is only a special case of the pre-wave with the steep plateau. At lower reactant concentrations the pre-wave and the main wave are merged usually. If the adsorption is weak, they can be merged at all concentrations.

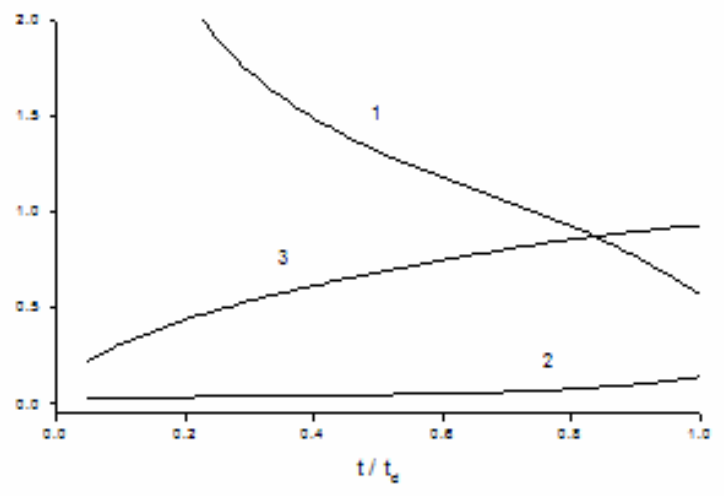

Figure 8. A change of $I / I_{L}(1),\left(c_{o x}\right)_{x=0} / c_{o x}^{*}$ (2) and $\theta$ (3) during the life-time of a single drop; $p=0.9, q=100, a=-3$ and $E-E^{0}=0.070 \mathrm{~V}$. 


\section{References}

1. J. Heyrovsky, J. Kuta. Principles of polarography, Academic Press, New York, 1966, p. 287.

2. Z. Galus, Fundamentals of electrochemical analysis, II ed., Ellis Horwood and Polish Scientific Publishers, New York, Warsaw, 1994, p. 481.

3. M. Zelić, M. Lovrić, Electrochim. Acta 35 (1990) 1701-1706.

4. $\quad$ M. Lovrić, Š. Komorsky-Lovrić, Langmuir 11 (1995) 1784-1790.

5. M. Lovrić, J. Electroanal. Chem. 465 (1999) 30-36.

6. J.E. Anderson, A.M. Bond, R.D. Jones, R.J. O'Halloran, J. Electroanal. Chem. 130 (1981) 113-122.

7. P. Delahay, I. Trachtenberg, J. Am. Chem. Soc. 79 (1957) 2355-2362. 